\title{
Placental growth factor gene silencing mitigates the epithelial-to-mesenchymal transition via the p38 MAPK pathway in rats with hyperoxia-induced lung injury
}

\author{
SHUANG ZHAO ${ }^{1}$, GANG LUO $^{2}$, HONGMIN WU $^{3}$ and LIANG ZHANG ${ }^{3}$ \\ ${ }^{1}$ Department of Pediatrics, The Fourth People's Hospital of Shenyang, Shenyang, Liaoning 110003; \\ Departments of ${ }^{2}$ Pediatrics and ${ }^{3}$ Neonatology, The First Affiliated Hospital of China Medical University, \\ Shenyang, Liaoning 110001, P.R. China
}

Received February 20, 2019; Accepted July 26, 2019

DOI: $10.3892 / \mathrm{mmr} .2019 .10785$

\begin{abstract}
Hyperoxia may cause pulmonary fibrosis in neonates and is characterized by the epithelial-to-mesenchymal transition (EMT) of alveolar epithelial cells. The placental growth factor (PLGF) gene is a member of the vascular endothelial growth factor family and is highly expressed in lung tissues that have been exposed to hyperoxia. The aim of the present study was to assess the role of PLGF in the EMT of lung tissue. Lung tissue exhibiting low PLGF expression was obtained by injecting rats exposed to hyperoxia with a PLGF-silencing lentiviral plasmid. Western blot analysis and immunohistochemistry revealed that expression levels of the EMT-related protein epithelial-cadherin were increased, whereas its inhibitor protein zinc-finger E-box binding homeobox 2 was decreased in these rats. These data demonstrated that PLGF silencing may significantly mitigate hyperoxia-induced EMT in rat lung tissue. Additionally, an increase in phosphorylated-p38 MAPK protein expression indicated that PLGF may be able to regulate hyperoxia-induced lung injury in rats via the p38 MAPK pathway.
\end{abstract}

\section{Introduction}

Recent advancements in neonatal intensive care units have led to an increase in the survival rates of the majority of early gestation premature infants; however, some of these develop bronchopulmonary dysplasia (BPD). BPD is one of the most common consequences of premature birth, affecting $~ 30 \%$ of infants with birth weights of $<1,500 \mathrm{~g}(1)$. With an increased

Correspondence to: Dr Liang Zhang, Department of Neonatology, The First Affiliated Hospital of China Medical University, 155 Nanjingbei Street, Shenyang, Liaoning 110001, P.R. China E-mail: lzhang@cmu.edu.cn

Key words: placental growth factor, hyperoxia-induced lung injury, epithelial-to-mesenchymal transition, p38 mitogen-activated protein kinase, bronchopulmonary dysplasia survival rate in infants with extremely low birth weights, BPD has increased in premature infants with a gestational age of $<28$ weeks (2). Infants exhibiting signs of BPD may require respiratory support following hospital discharge and may experience chronic respiratory morbidity throughout their whole lives; they are at higher risk of neurodevelopment problems and mortality (3). Infants and children with a history of BPD may require re-admission to hospital repeatedly in their early years, with some developing severe lung function abnormalities, including asthma, chest deformities and adolescent pulmonary hypertension. Delayed neurodevelopment can cause cognitive and motor function defects, such as cerebral palsy, which is common in BPD infants (4). Currently, there are a lack of effective treatments options for BPD. BPD is characterized by the arrest of alveolar development and is secondary to myofibroblast-mediated extracellular matrix deposition and fibrotic lung formation (5). Type II alveolar epithelial cells (AECIIs) serve an important role in the development of BPD. Hyperoxia can induce AECIIs to differentiate into fibroblasts and affect alveolar development through the epithelial-to-mesenchymal (EMT) transition (6).

EMT is the process in which epithelial cells lose their polarity and cell junctions, and acquire the characteristics of mesenchymal cells (7). During BPD, AECII cells undergo EMT, which promotes the progression of pulmonary fibrosis (8). SMAD-dependent (SMAD2 and SMAD3) and non-dependent cellular signaling pathways [PI3K/AKT, RhoA, partitioning defective 6 homolog and mitogen-activated protein kinase (MAPK)] have been reported to be associated with EMT, and it has been previously demonstrated that bleomycin-induced lung fibrosis in mice can be alleviated by the p38 MAPK protein inhibitorFR-167653 (9). Epithelial cell (E)-cadherin, an important member of the cadherin family, is widely distributed in a variety of epithelial cells. E-cadherin is important for epithelial cell adhesion, the maintenance of epithelial tissue integrity and polarity. The downregulation of E-cadherin is associated with the development of EMT (10). The loss of E-cadherin expression in the epithelia is considered a hallmark of EMT (11). Zinc-finger E-box binding homeobox 2 (ZEB2) has also been demonstrated to be an important regulatory molecule in EMT; the upregulation of ZEB2 can inhibit 
E-cadherin transcription and induce EMT, which may lead to cell invasion and increased malignancy (12).

Vascular endothelial growth factors (VEGFs) are a family of essential angiogenic mediators that serve important roles in the regulation of angiogenesis. Placental growth factor (PLGF) and VEGFA are two important molecules in this family (13). PLGF was first identified in placental tissue, and subsequent studies have demonstrated that it is expressed in numerous tissues types, including heart, lung and thyroid (14-17). In breast cancer cells, PLGF has been indicated to promote the progression of EMT (18). In cervical cancer cells, PLGF has been reported to be associated with the process of EMT, allowing cancer cells to leave the primary tumor, invade surrounding tissues and spread to the distal organ (19). Our previous study reported that PLGF expression is increased in lungs exposed to hyperoxia (20). It was therefore hypothesized that PLGF may be associated with lung injury through the promotion of EMT. To address this hypothesis, PLGF gene silencing was used in neonatal rat lung tissue to investigate the underlying mechanisms by which this may occur, which provided valuable information regarding disease pathogenesis and potential therapeutic approaches for BPD.

\section{Materials and methods}

Materials. A total of 32 14-day old Sprague-Dawley (SD) rats (female:male, 17:15; 25.2-30.3 g) were provided by The Experimental Animal Center of China Medical University. The animals were housed at a temperature of $25-27^{\circ} \mathrm{C}$, with a humidity of $50-70 \%$ and a $12 \mathrm{~h}$ light/dark cycle with ad libitum access to food and water. Xylene, absolute ethanol, eosin Y and hydrogen peroxide were purchased from Wuhan USCN Business Co., Ltd. Hematoxylin, eosin and goat serum (cat. no. SL038) were purchased from Beijing Solarbio Science\& Technology Co., Ltd. PLGF mouse monoclonal antibody (cat. no. sc-518003) and E-cadherin mouse monoclonal antibody (cat. no. sc-71007) were purchased from Santa Cruz Biotechnology, Inc. Anti-phosphorylated (p)-p38 rabbit polyclonal antibody (cat. no. bs-2210R) was purchased from (BIOSS). Anti-p38 rabbit monoclonal (cat. no. M00176), anti- $\beta$-actin goat polyclonal (cat. no. BM0627) and anti-ZEB2 rabbit polyclonal (cat. no. PA1959) antibodies were purchased from Boster Biological Technology. Biotin-labeled goat anti-mouse or goat anti-rabbit IgG and HRP-labeled streptavidin (cat. nos. A0286; A0277; A0303, respectively) were purchased from Beyotime Institute of Biotechnology. RIPA lysis buffer was purchased from Tiangen Biotech Co., Ltd. BCA Protein Assay Reagent kit was obtained from Pierce (Thermo Fisher Scientific, Inc.). TRIzol was obtained from Thermo Fisher Scientific, Inc.

Animal experiments. Neonatal SD rats, delivered prematurely at 21 days gestation from the rats detailed above, were placed in an oxygen chamber with their mothers. Oxygen was continuously supplied to maintain fraction of inspired oxygen $\left(\mathrm{FiO}_{2}\right)=75 \%$ (hyperoxic condition; ProOx $110 \mathrm{O}_{2}$ Controller; BioSpherix, Ltd.), a $\mathrm{CO}_{2}$ concentration of $<0.5 \%$ (sodium lime absorption $\mathrm{CO}_{2}$ ), a temperature of $22-27^{\circ} \mathrm{C}$ and a humidity of $50-70 \%$. Normoxic conditions were identical to hyperoxia except $\mathrm{FiO}_{2}=21 \%$. Chambers were opened for 30 min every day to add water, feed and replace the litter. Mother rats were alternated between hyperoxia and normoxia conditions to prevent oxygen toxicity and to provide equal nutrition. The neonatal rats (8/group) were randomly divided into: i) Normoxia control group $\left(\mathrm{FiO}_{2}=21 \%\right)$; ii) hyperoxia group $\left(\mathrm{FiO}_{2}=75 \%\right)$; iii) hyperoxia + negative control (NC) lentivirus group (hyperoxia + shRNA-NC); and iv) hyperoxia + short hairpin (sh)RNA-PLGF lentivirus group (hyperoxia + shRNA-PLGF). After being anesthetized by intraperitoneal injection with pentobarbital (30-40 mg/kg) (Tianjin Kemiou Chemical Reagent Co., Ltd.), rat pups were sacrificed after 14 days of exposure (8/group).

Lentiviral PLGF interference plasmid injection. The $72 \mathrm{bp}$ oligonucleotide short hairpin (sh)RNAs specific for PLGF were obtained from Shanghai GenePharma Co., Ltd. The sequences of PLGF shRNAs were as follows: 5'-GCGCTA AAGACAGCCAACA-3'. Non-targeting shRNA, with a sequence of 5'-TTCTCCGAACGTGTCACGT-3', was used as a negative control. The PLGF shRNAs were sub-cloned into a lentiviral vector (GV248; Shanghai GeneChem Co., Ltd.). Following this, the lentiviral particles with the shRNA-PLGF were obtained from Shanghai GeneChem Co., Ltd and were directly injected into the neonates via tail-intravenous injection at a concentration of $3 \times 10^{8} \mathrm{TU} / \mathrm{kg}$, every day for 3 consecutive days. PLGF expression in the lung tissue was determined using western blot analysis.

Immunohistochemistry. Lung tissues were harvested from the neonates, fixed in $10 \%$ formalin at $37^{\circ} \mathrm{C}$ for $48 \mathrm{~h}$ and imbedded in paraffin. The specimens were then cut into $5 \mu \mathrm{m}$ sections, which were deparaffinized with xylene and rehydrated in a descending ethanol series $(95,85$ and $75 \%)$, underwent antigen retrieval with $1 \%$ sodium citrate buffer at $100^{\circ} \mathrm{C}$ for $20 \mathrm{~min}$, followed by incubation with $3 \%$ hydrogen peroxide and blocking with $10 \%$ goat serum for $30 \mathrm{~min}$, both at room temperature. Sections were incubated overnight at $4^{\circ} \mathrm{C}$ with primary antibodies against PLGF (1:200) and E-cadherin (1:50), followed by incubation a corresponding biotin-labelled secondary antibody $(1: 200)$ at $37^{\circ} \mathrm{C}$ for $30 \mathrm{~min}$ with. Sections were then incubated with DAB and counterstained with hematoxylin. Slides were examined using a light microscope and images were captured at x400 magnification. Cells exhibiting brown-yellow particles in the cytoplasm were regarded as positive-stained cells.

Hematoxylin and eosin $(H \& E)$ staining. The lung tissues sections embedded in paraffin were cut into $4 \mu \mathrm{m}$ thick sections. After deparaffinization and rehydration, the sections were stained using H\&E at room temperature for $15 \mathrm{~min}$. Images were captured at x200 magnification using a light microscope and changes in lung tissue morphology were recorded.

Western blot analysis. Lung tissue $\left(\sim 1 \mathrm{~cm}^{3}\right)$ was disrupted using sonication $\left(50 \mathrm{kHz}\right.$ at $4^{\circ} \mathrm{C}$ for $\left.15 \mathrm{~min}\right)$, liquid nitrogen grinding and RIPA lysis buffer protein extraction. Total protein was quantified using the BCA method. Equal amounts of protein $(20 \mu \mathrm{g})$ were separated using $8 \%$ SDS-PAGE and transferred to PVDF membranes. The membranes were blocked at room temperature for $2 \mathrm{~h}$ using $5 \%$ skim milk powder. After blocking, the membranes were incubated overnight at $4{ }^{\circ} \mathrm{C}$ with primary antibodies (all 1:500) against PLGF, E-cadherin, ZEB2, p38, phosphorylated (p)-p38, and 
A
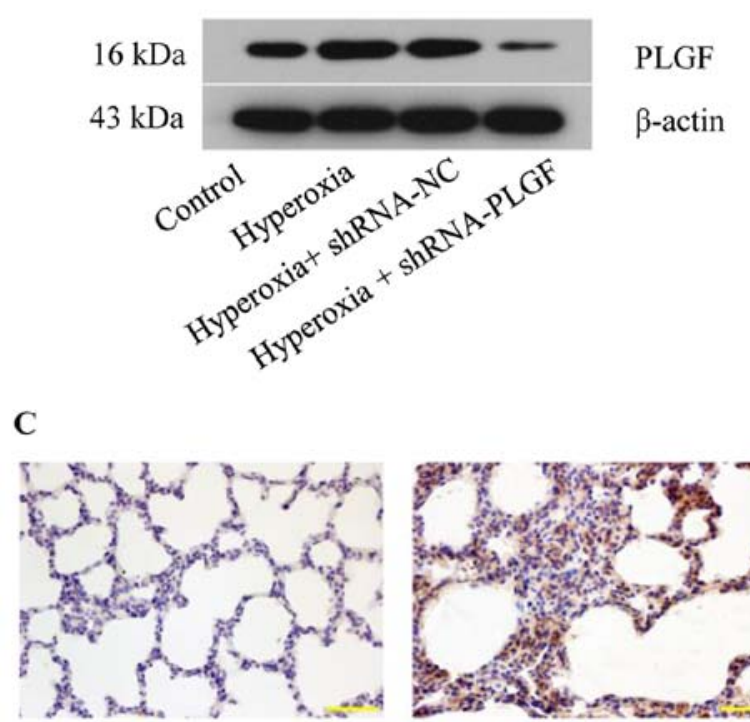

Control

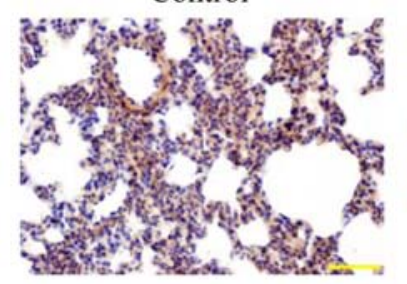

Hyperoxia + shRNA-NC

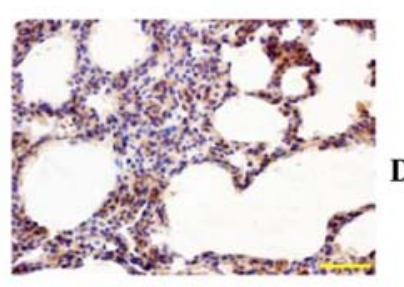

Hyperoxia

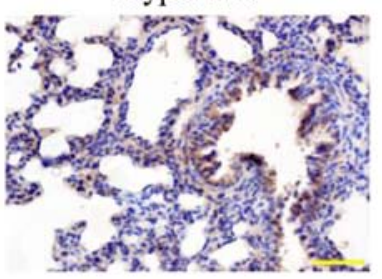

Hyperoxia + shRNA-PLGF
B

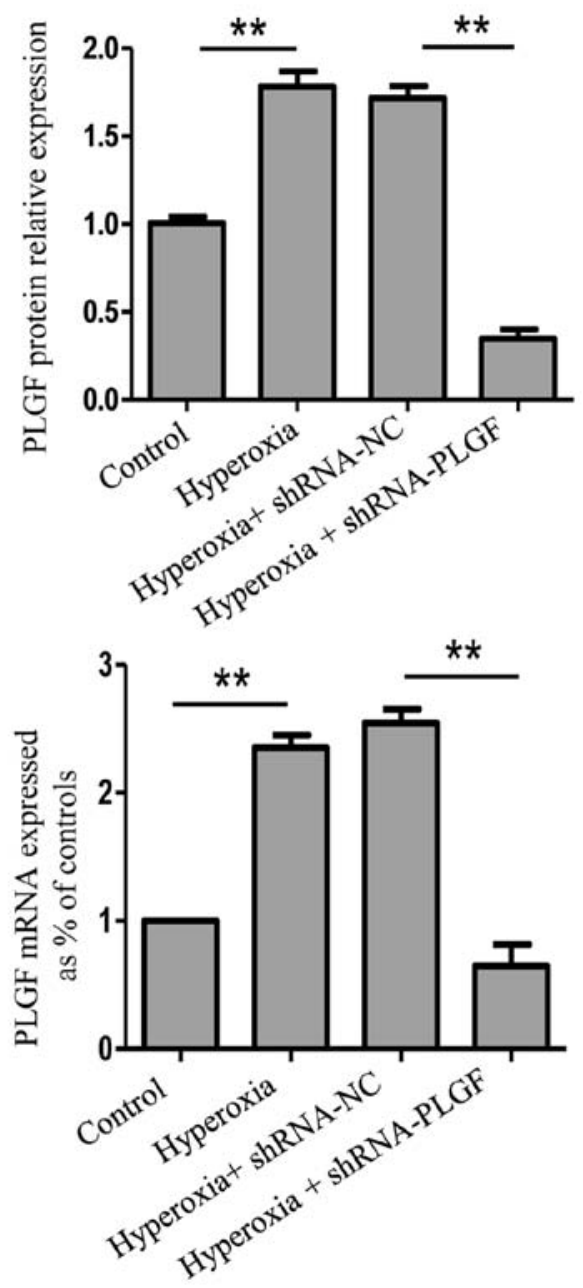

Figure 1. Expression of PLGF in hyperoxia-exposed lung tissue. Neonatal rats were exposed to normoxic or hyperoxic conditions for 14 days; hyperoxia exposed rats were subsequently injected with shRNA-NC or shRNA-PLGF. (A) Western blot analysis and (B) quantification was performed to determine PLGF protein expression levels in rat lung tissues; $\beta$-actin was used as the endogenous control. Data are presented as the mean \pm standard deviation. $\mathrm{n}=8$. ${ }^{* *} \mathrm{P}<0.01$. (C) Immunostaining of rat lung tissues for PLGF expression; hematoxylin was used as a counterstain. Magnification, $\mathrm{x} 100$; scale bar, $100 \mu \mathrm{m}$. (D) PLGF mRNA expression levels were detected by reverse transcription-quantitative PCR. Data are presented as the mean \pm standard deviation. $\mathrm{n}=3$. ${ }^{* *} \mathrm{P}<0.01$. NC, negative control; PLGF, placental growth factor; shRNA, short hairpin RNA.

subsequently incubated with HRP-conjugated secondary antibodies $(1: 1,000)$ at room temperature for $1 \mathrm{~h}$ and developed using ECL Plus western blotting detection reagents. ImageJ 1.8.0 (National Institutes of Health) was used to analyze band density. $\beta$-actin $(1: 5,000)$ was used as a loading control and to normalize protein expression.

Reverse transcription-quantitative PCR (RT-qPCR). Total RNA was isolated from $1 \mathrm{~cm}^{3}$ lung tissue using TRIzol ${ }^{\circledR}$ (Invitrogen; Thermo Fisher Scientific, Inc.), according to the manufacturer's protocol, and RT was performed using $1 \mu \mathrm{g}$ total RNA using the PrimeScript ${ }^{\mathrm{TM}}$ RT Master Mix (Takara Bio, Inc.). The protocol was: At $25^{\circ} \mathrm{C}$ for $10 \mathrm{~min}$ followed by incubation at $42^{\circ} \mathrm{C}$ for $50 \mathrm{~min}$. The reaction was inactivated by heating at $70^{\circ} \mathrm{C}$ for $15 \mathrm{~min}$.

qPCR was performed using an ABI PRISM 7500HT System (Applied Biosystems; Thermo Fisher Scientific, Inc.) and the TB Green Premix Ex Taq II kit (Takara Bio, Inc.) according to the manufacturer's protocol. The relative gene expression was calculated using the $2^{-\Delta \Delta \mathrm{Cq}}$ method (21), normalized to the housekeeping gene GAPDH. The primers used were as follows:
PLGF, forward 5'-CCCACCTGGATGCTGTT-3', reverse 5'-ATAGAGGGTAGGTACCAGCA-3'; E-cadherin, forward 5'-ACTTTGGTGTGGGTCTGGAG-3', reverse 5'-TCTGTG GCAATGATGAGAGC-3'; ZEB2, forward 5'-TGATTGAGA ACCACAGCATACC-3', reverse 5'-GTTCATCAGAGTTGG GTTCCAT-3'; GAPDH, forward 5'-GCACCGTCAAGGCTG AGAAC-3', reverse 5'-TGGTGAAGACGCCAGTGGA-3'.

Statistical analysis. GraphPad Prism version 8.0 (GraphPad Software, Inc.) was used to analyze the data. Pairwise comparisons were performed using the Student's t-test method. Two-way ANOVA followed by Student-Newman-Keuls pot hoc test was used to compare multiple groups. All experiments were repeated at least three times. Data are presented as the mean \pm standard deviation. $\mathrm{P}<0.05$ was considered to indicate a statistically significant difference.

\section{Results}

PLGF expression in hyperoxic lung tissue after PLGF gene silencing. Following lentiviral plasmid injection, PLGF 
A

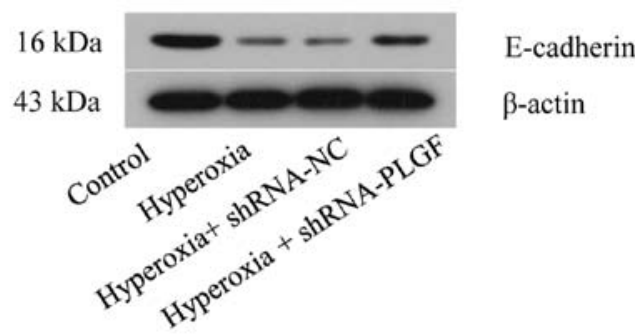

B
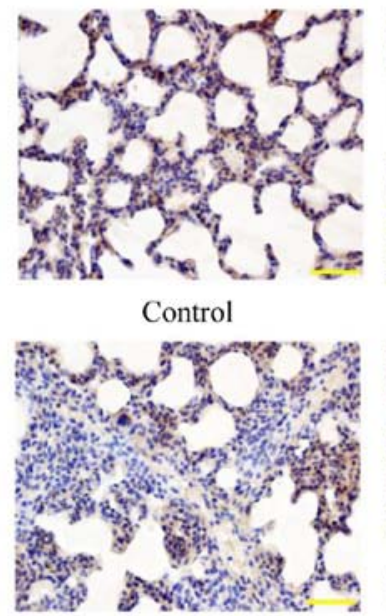

Hyperoxia + shRNA-NC

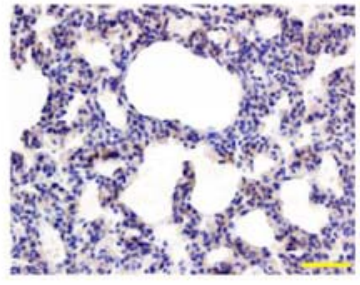

Hyperoxia

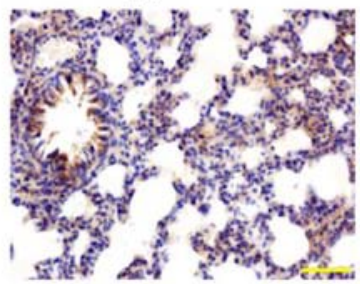

Hyperoxia + shRNA-PLGF
C
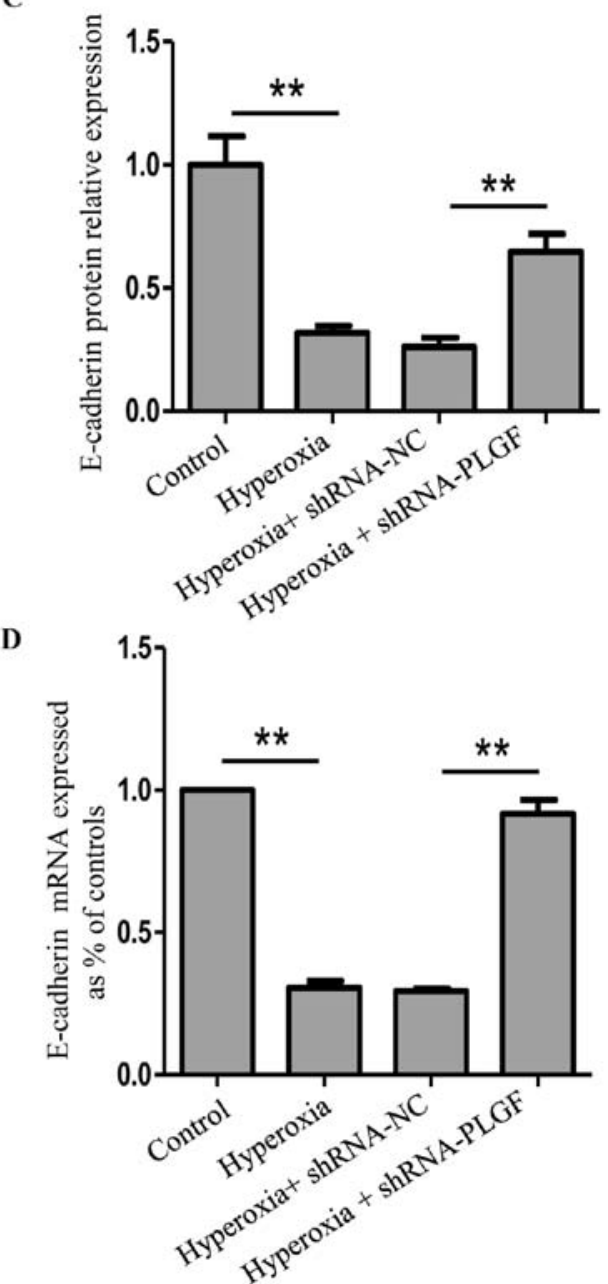

Figure 2. Expression of E-cadherin in hyperoxia-exposed lung tissue. Neonatal rats were exposed to normoxic or hyperoxic conditions for 14 days; hyperoxia exposed rats were subsequently injected with shRNA-NC or shRNA-PLGF. (A) Western blot analysis and (B) quantification was performed to determine E-cadherin protein expression levels in rat lung tissues; $\beta$-actin was used as the endogenous control. Data are presented as the mean \pm standard deviation. $\mathrm{n}=8$. ${ }^{* *} \mathrm{P}<0.01$. (C) Immunostaining of rat lung tissues for E-cadherin expression; hematoxylin was used as a counterstain. Magnification, $\mathrm{x} 100$; scale bar, $100 \mu \mathrm{m}$. (D) E-cadherin mRNA expression levels were detected by reverse transcription-quantitative PCR. Data are presented as the mean \pm standard deviation. $\mathrm{n}=3$. ${ }^{* *} \mathrm{P}<0.01$. E-cadherin, epithelial cadherin; NC, negative control; PLGF, placental growth factor; shRNA, short hairpin RNA.

expression was determined using western blot analysis and immunohistochemistry 14 days after the first injection (Fig. 1). Compared with the normoxia control group, PLGF protein expression levels in rat lung tissue were significantly increased following exposure to hyperoxia (Fig. 1A and B). A similar change was detected at the mRNA level (Fig. 1D). Immunohistochemical analysis revealed a large number of PLGF-positive cells in the hyperoxia group compared with the normoxia control group (Fig. 1C). In the hyperoxia + shRNA-NC group, western blotting analysis revealed that the PLGF protein expression level in the lung tissue was also significantly increased compared with the normoxia group, and similar results were observed in the immunohistochemistry experiments. In the hyperoxia + shRNA-PLGF group, lung tissue PLGF protein expression levels were significantly lower compared with the hyperoxia + shRNA-NC group, and the number of positively stained cells decreased, as determined using immunohistochemistry (Fig. S1). These data demonstrated that hypoxia induced an increase in the expression of PLGF in lung tissues and that PLGF gene expression was
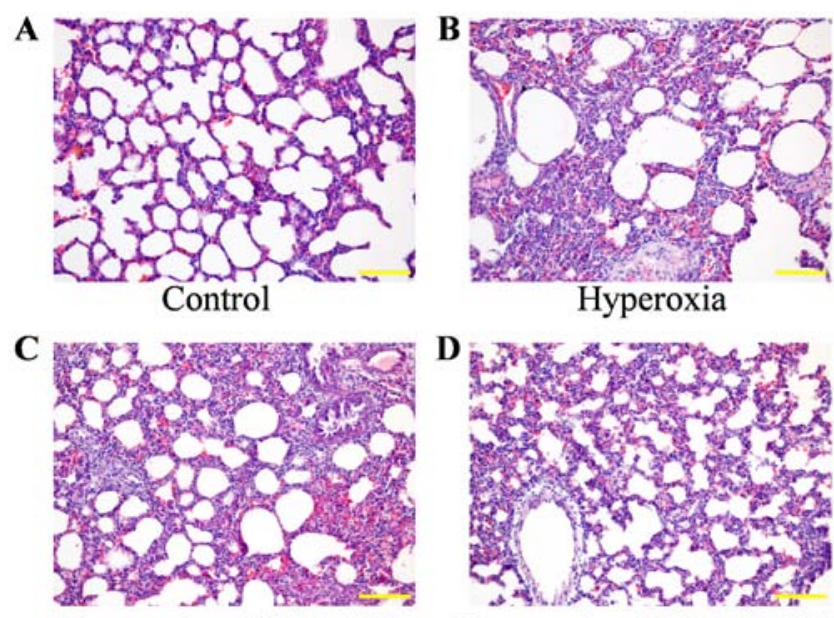

Hyperoxia+shRNA-NC

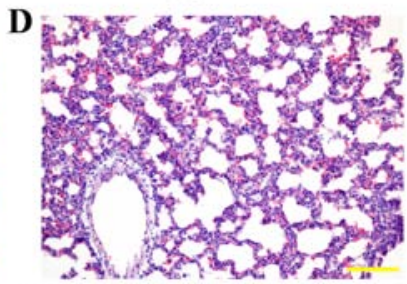

Hyperoxia+shRNA-PLGF

Figure 3. Hematoxylin and eosin staining of rat hypoxia-exposed lung tissues. Neonatal rats were exposed to (A) normoxic or (B) hyperoxic conditions for 14 days; hyperoxia exposed rats were subsequently injected with (C) shRNA-NC or (D) shRNA-PLGF. Magnification, x100; scale bar, $100 \mu \mathrm{m}$. NC, negative control; PLGF, placental growth factor; shRNA, short hairpin RNA. 
A

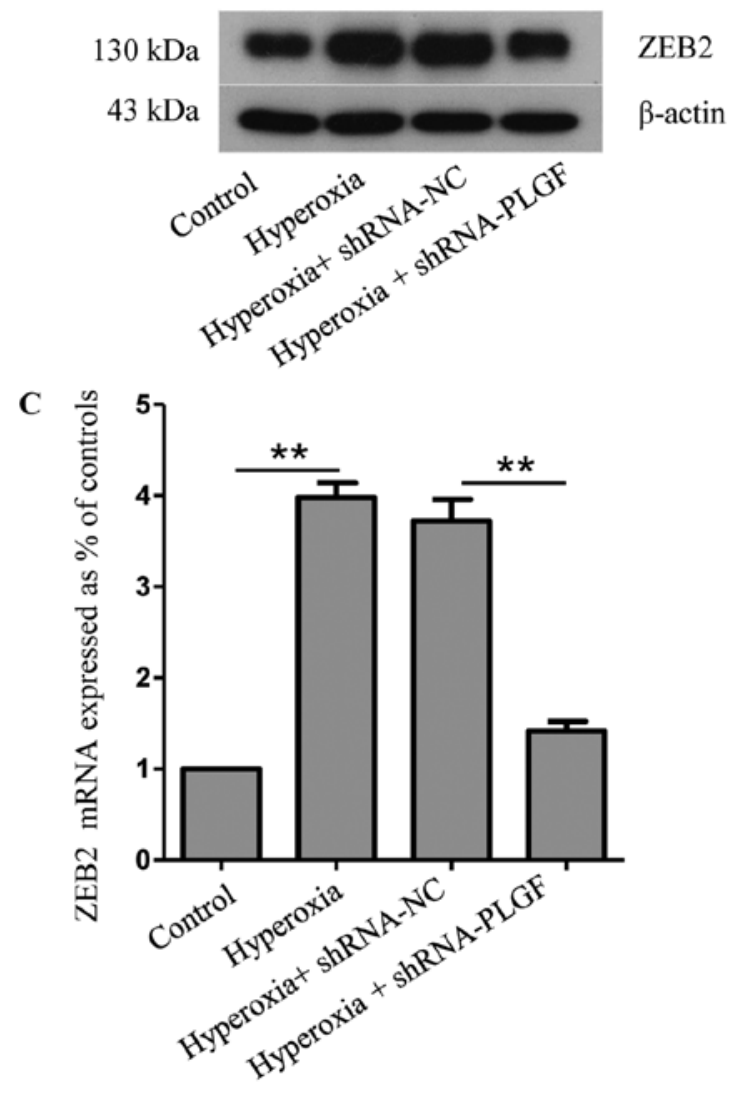

B

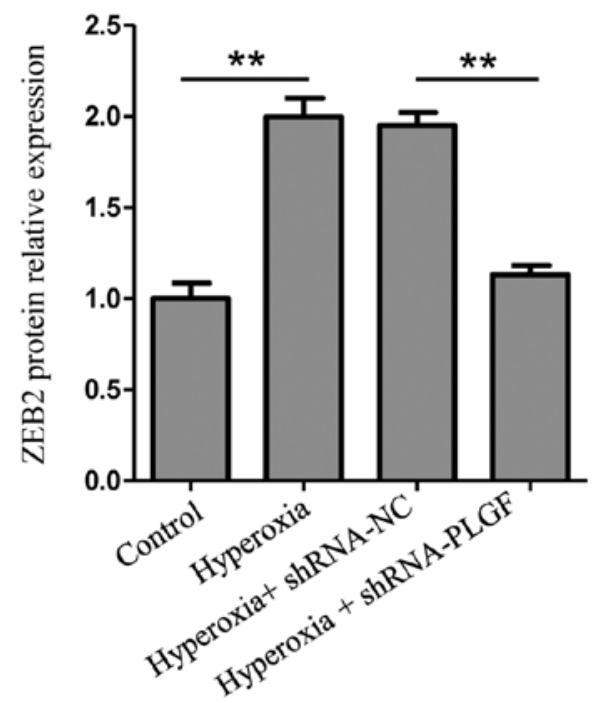

Figure 4. Expression of ZEB2 in hyperoxia-exposed lung tissue. Neonatal rats were exposed to normoxic or hyperoxic conditions for 14 days; hyperoxia exposed rats were subsequently injected with shRNA-NC or shRNA-PLGF. (A) Western blot analysis and (B) quantification was performed to determine ZEB2 protein expression levels in rat lung tissues; $\beta$-actin was used as the endogenous control. Data are presented as the mean \pm standard deviation. $n=8$. ${ }^{* *} \mathrm{P}<0.01$. (C) ZEB2 mRNA expression levels were detected by reverse transcription-quantitative PCR. Data are presented as the mean \pm standard deviation. $\mathrm{n}=3$. $^{* *} \mathrm{P}<0.01$. NC, negative control; PLGF, placental growth factor; shRNA, short hairpin RNA; ZEB, zinc-finger E-box binding homeobox 2 .

successfully silenced following lentiviral interference plasmid injection in rats.

E-cadherin expression in hyperoxic lung tissue after PLGF gene silencing. The effects of PLGF gene silencing on E-cadherin protein expression in the lung tissue of hyperoxia-exposed rats was determined using western blot, RT-qPCR and immunohistochemistry (Fig. 2). Compared with the normoxia control group, E-cadherin protein expression in lung tissue following hyperoxia treatment was significantly decreased (Fig. 2A and B). In the hyperoxia + shRNA-NC group, E-cadherin expression also significantly decreased compared with the normoxia group; no significant difference was identified in comparison with the hyperoxia group. In hyperoxia + shRNA-PLGF rats, E-cadherin protein expression in lung tissue was significantly increased compared with the hyperoxia + shRNA-NC group (Fig. 2A and B). The number of positively stained cells was also increased in the hyperoxia + shRNA-PLGF group compared with the hyperoxia + shRNA-NC group, as determined using immunohistochemistry (Fig. 2C). These results indicated that hyperoxia may induce EMT, as determined by the decreased E-cadherin expression, and that shRNA-PLGF injection may delay this EMT in the lung tissue of rats exposed to hyperoxia.
Pathological effects of PLGF gene silencing on lung tissue in rats with hyperoxia-induced lung injury. The pathological effect of PLGF gene silencing on hyperoxia-induced lung injury rats was observed using H\&E staining (Fig. 3). Compared with the control group, the alveolar epithelium was swollen in the hyperoxia group, large amounts of exudate were present in the alveolar space and alveolar structure was simplified. The lung tissue of rats in the hypoxia + shRNA-NC group exhibited similar results to the hyperoxia group, with the alveolar epithelium being highly swollen and structurally simplified. In hyperoxia rats injected with the shRNA-PLGF, the abnormal epithelial structure was lessened and the lungs exhibited features, including a reduction in alveolar exudate, a clear alveolar structure and alleviated interstitial edema.

Expression of ZEB2 and p-38MAPK/p-p38MAPK after PLGF gene silencing. ZEB2 is closely related to, and is a specific antagonist of, E-cadherin; both serve important roles in EMT (22). Compared with the normoxia control group, ZEB2 protein and mRNA expression levels in rat lung tissue was significantly increased in the hyperoxia group (Fig. 4A-C). ZEB2 expression in the lung tissue of hyperoxia + shRNA-NC rats was also significantly increased compared with the normoxia group. Compared with the hyperoxia + shRNA-NC group, ZEB2 protein and mRNA expression levels in the lung 
$\mathbf{A}$

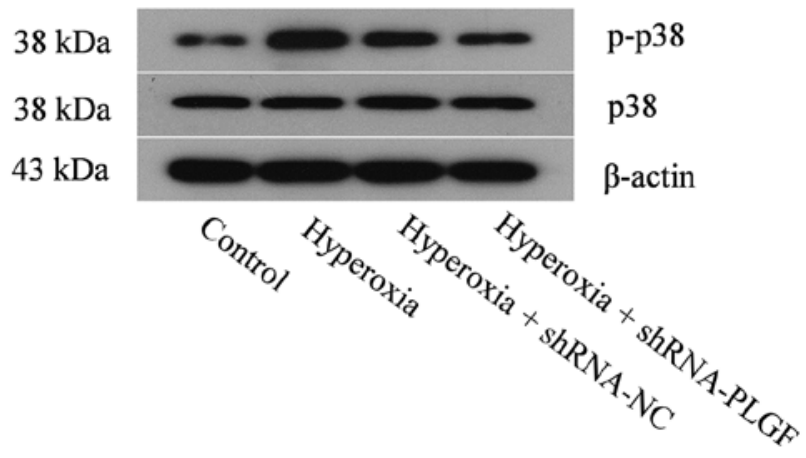

B

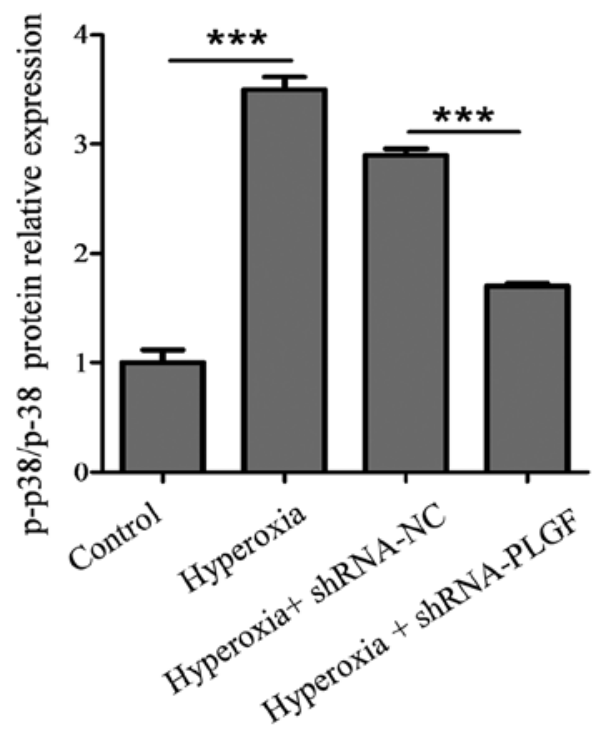

Figure 5. Expression of p38 and p-p38 MAPK in hyperoxia-exposed lung tissue. Neonatal rats were exposed to normoxic or hyperoxic conditions for 14 days; hyperoxia exposed rats were subsequently injected with shRNA-NC or shRNA-PLGF. (A) Western blot analysis and (B) quantification was performed to determine p-p38/p38 protein expression levels in rat lung tissues; $\beta$-actin was used as the endogenous control. Data are presented as the mean \pm standard deviation. $n=8 .{ }^{* * *} \mathrm{P}<0.001$. MAPK, mitogen-activated protein kinase; NC, negative control; PLGF, placental growth factor; shRNA, short hairpin RNA.

tissue of hyperoxia + shRNA-PLGF rats was significantly decreased (Fig. 4A-C). These data suggested that hyperoxia may cause EMT in rat lung tissue, whereas PLGF gene silencing can reduce this effect (Fig. 4).

p38 protein kinase is a key factor in the MAPK signaling pathway and is closely associated with the activation of $\mathrm{NF}-\kappa \mathrm{B}$ pathways, which can induce EMT in the lungs (23). Compared with the normoxia control group, p-p38 expression in the lung tissue of hyperoxia rats was significantly increased (Fig. 5). In the hyperoxia + shRNA-NC rats, p-p38 expression was also significantly increased compared with the control group. p-p38 expression in lung tissue of hyperoxia + shRNA-PLGF rats was significantly decreased compared with the hyperoxia + shRNA-NC group. Although the treatment groups were different, the p38 content did not change significantly.

\section{Discussion}

In the present study, evidence of the role of PLGF in the acute stage of hyperoxia-induced lung injury was presented in the neonatal rat. The present study aimed to determine the pathogenesis and the molecular mechanisms behind BPD with severe lung function abnormalities. A previous study indicated the molecular basis of lung injury using cultured lung cells in vitro (24). In the present study, the role of PLGF in hyperoxia-induced pulmonary dysplasia in vivo was investigated using the neonatal rat disease model of hyperoxia-induced lung injury.

Previous studies have demonstrated that PLGF can induce EMT in a variety of disease, including cervical cancer and breast cancer $(18,19)$, and it has also been reported that hyperoxia can promote EMT in alveolar cells (25). In concurrence with these previous findings, results from the present study demonstrated that PLGF and ZEB2 expression levels increased, whereas E-cadherin expression decreased in hyperoxia exposed neonatal rats. To confirm these results, a
PLGF-silencing lentiviral plasmid was used to reduce PLGF expression in hyperoxia-exposed neonatal rat lungs; the results demonstrated that E-cadherin expression was increased and ZEB2 expression was decreased. These results indicated that PLGF may serve a role in regulating EMT in neonatal rat lung tissue during exposure to hyperoxia.

The p38 MAPK signal transduction pathway is present in the majority of cells. This pathway transduces extracellular stimuli into cells and their nuclei, and causes the induction of cellular biological reactions including cell proliferation, differentiation, transformation and apoptosis (26). The activation of the p38 MAPK signaling pathway promotes NF- $\kappa$ B inhibitor $\alpha$ phosphorylation and degradation and activates the $N F-\kappa B$ pathway. This suggests that $\mathrm{p} 38$ MAPK may induce pulmonary fibrosis by activating NF- $\kappa \mathrm{B}$ (27). In our previous study, it was demonstrated that NF- $\kappa \mathrm{B}$ increased during hyperoxia (28). To determine if $\mathrm{p} 38$ MAPK served a role in the process of EMT, p-p38MAPK expression was determined in neonatal rat lungs in the present study. The results indicated that PLGF and p-p38MAPK expression increased in neonatal rat lungs during hyperoxia. PLGF silencing resulted in the reduced expression of p-p38MAPK. These results suggested that the p38 MAPK pathway may be associated with the regulation of the EMT process in neonatal rat lung tissue during hyperoxia. Previous studies have suggested that ERK activation in lung cells has a protective effect in response to hyperoxia $(29,30)$, through stimulation of DNA repair and antioxidant mechanisms, and prolonged cell survival. Conversely, JNK1/2 and p38 kinase have been most frequently reported to have roles in induction of apoptotic responses (31). The present study indicated that PLGF may be able to regulate hyperoxia-induced lung injury in rats via the p38 MAPK pathway.

It had been reported that PLGF promoted migration through regulating EMT-related protein expression in cervical cancer (19). In the presented study, the activation of p38 in hyperoxia-induced lung tissue was demonstrated, which may 
have harmful effects on the lung cells. It is possible that the different types of cells have the different ability of resistance to injury. Further investigation will be done using the alveolar epithelial cell lines such as RLE-6TN to better dissect the role of the signaling axis during hyperoxia-induced lung injury.

A previous study has revealed that PLGF expression increased in hyperoxia-exposed primary AECIIs (24), which contributed to hyperoxia-induced lung injury through the promotion of apoptosis and EMT. These data suggested that PLGF may be a potential therapeutic target for hyperoxia-induced lung injury. In the present study, the role of PLGF in hyperoxia-induced pulmonary dysplasia was examined using a neonatal SD rat model, with a focus on the alterations of lung tissue in vivo. To further investigate the role of PLGF in hyperoxia-induced pulmonary injury, lentiviral plasmids were used to silence the PLGF gene and the effects on lung injury were examined in vivo. These results indicated that PLGF knockdown in vivo may attenuate lung tissue injury under hyperoxia, which was predominantly due to the depletion of PLGF inhibiting p38 MAPK-mediated EMT. Additionally, treating cultured lung cells with an ERK inhibitor, such as PD98059, will be necessary to delineate the signaling axis in future studies.

In summary, the results of the present in vivo study further supported previous in vitro findings, which suggested that the reduction of PLGF, using RNA interference-based gene silencing, maybe a potential method to use to reduce hyperoxia-induced lung injury.

\section{Acknowledgements}

Not applicable.

\section{Funding}

The present study was supported by the National Science Foundation of Liaoning (grant no. 20180530094).

\section{Availability of data and materials}

The datasets used and/or analyzed during the present study are available from the corresponding author on reasonable request.

\section{Authors' contributions}

SZ and LZ designed the study. SZ and GL performed the experiments. HMW and $\mathrm{LZ}$ were involved in the statistical analyses. SZ and LZ wrote and revised the manuscript. All authors read and approved the final manuscript, and all authors confirm its accuracy.

\section{Ethics approval and consent to participate}

Ethical approval for the present study was provided by China Medical University Ethics Committee (Shenyang, China).

\section{Patient consent for publication}

Not applicable.

\section{Competing interests}

The authors declare that they have no competing interests.

\section{References}

1. Jobe AH: The new bronchopulmonary dysplasia. Curr Opin Pediatr 23: 167-172, 2011.

2. Stoll BJ, Hansen NI, Bell EF, Walsh MC, Carlo WA, Shankaran S, Laptook AR, Sánchez PJ, Van Meurs KP, Wyckoff M, et al: Trends in care practices, morbidity, and mortality of extremely preterm neonates, 1993-2012. JAMA 314: 1039-1051, 2015.

3. Greenough A: Long term respiratory outcomes of very premature birth (<32 weeks). Semin Fetal Neonatal Med 17: 73-76, 2012.

4. Kinsella JP, Greenough A and Abman SH: Bronchopulmonary dysplasia. Lancet 367: 1421-1431, 2006.

5. Li J, Li Y, He H, Liu C, Li W, Xie L and Zhang Y: Csk/Src/EGFR signaling regulates migration of myofibroblasts and alveolarization. Am J Physiol Lung Cell Mol Physiol 310: L562-L571, 2016.

6. Yang H, Fu J, Xue X, Yao L, Qiao L, Hou A, Jin L and Xing Y: Epithelial-mesenchymal transitions in bronchopulmonary dysplasia of newborn rats. Pediatr Pulmonol 49: 1112-1123, 2014.

7. Lamouille S, Xu J and Derynck R: Molecular mechanisms of epithelial-mesenchymal transition. Nat Rev Mol Cell Biol 15: 178-196, 2014.

8. Song JS, Kang CM, Park CK, Yoon HK, Lee SY, Ahn JH and Moon HS: Inhibitory effect of receptor for advanced glycation end products (RAGE) on the TGF- $\beta$-induced alveolar epithelial to mesenchymal transition. Exp Mol Med 43: 517-524, 2011.

9. Matsuoka H, Arai T, Mori M, Goya S, Kida H, Morishita H, Fujiwara H, Tachibana I, Osaki T and Hayashi S: A p38 MAPK inhibitor, FR-167653, ameliorates murine bleomycin-induced pulmonary fibrosis. Am J Physiol Lung Cell Mol Physiol 283: L103-L112, 2002.

10. Feldkoren B, Hutchinson R, Rapoport Y, Mahajan A and Margulis V: Integrin signaling potentiates transforming growth factor-beta 1 (TGF- $\beta 1$ ) dependent down-regulation of E-Cadherin expression-important implications for epithelial to mesenchymal transition (EMT) in renal cell carcinoma. Exp Cell Res 355: 57-66, 2017.

11. Serrano-Gomez SJ, Maziveyi M and Alahari SK: Regulation of epithelial-mesenchymal transition through epigenetic and post-translational modifications. Mol Cancer 15: 18, 2016.

12. Vandewalle C, Van Roy F and Berx G: The role of the ZEB family of transcription factors in development and disease. Cell Mol Life Sci 66: 773-787, 2009.

13. Andraweera PH, Dekker GA and Roberts CT: The vascular endothelial grow th factor family in adverse pregnancy outcomes. Hum Reprod Update 18: 436-457, 2012.

14. Hayes Ryan D, McCarthy FP, O'Donoghue K and Kenny LC: Placental growth factor: A review of literature and future applications. Pregnancy Hypertens 14: 260-264, 2018.

15. Iwasaki H, Kawamoto A, Tjwa M, Horii M, Hayashi S, Oyamada A, Matsumoto T, Suehiro S, Carmeliet P and Asahara T: PIGF repairs myocardial ischemia through mechanisms of angiogenesis, cardioprotection and recruitment of myo-angiogenic competent marrow progenitors. PLoS One 6: e24872, 2011.

16. Tsao PN, Li H, Wei SC, Ko ML, Chou HC, Hsieh WS and Hsieh FJ: Expression of angiogenic factors and their receptors in postnatal mouse developing lung. J Formos Med Assoc 103: 137-143, 2004.

17. Korevaar TI, Steegers EA, de Rijke YB, Visser WE, Jaddoe VW, Visser TJ, Medici M and Peeters RP: Placental angiogenic factors are associated with maternal thyroid function and modify hCG-mediated FT4 stimulation. J Clin Endocrinol Metab 100: E1328-E1334, 2015.

18. Ning Q, Liu C, Hou L, Meng M, Zhang X, Luo M, Shao S, Zuo X and Zhao X: Vascular endothelial growth factor receptor-1 activation promotes migration and invasion of breast cancer cells through epithelial-mesenchymal transition. PLoS One 8: e65217, 2013.

19. Huang W, Zhu S, Liu Q, Li C and Li L: Placenta growth factor promotes migration through regulating epithelial-mesenchymal transition-related protein expression in cervical cancer. Int J Clin Exp Pathol 7: 8506-8519, 2014.

20. Zhang L, Zhao S, Yuan L, Wu H, Jiang H and Luo G: Placenta growth factor contributes to cell apoptosis and epithelial-to-mesenchymal transition in the hyperoxia-induced acute lung injury. Life Sci 156: 30-37, 2016. 
21. Livak KJ and Schmittgen TD: Analysis of relative gene expression data using real-time quantitative PCR and the 2(-Delta Delta C(T)) method. Methods 25: 402-408, 2001

22. Zhu GJ, Song PP,Zhou H, Shen XH, Wang JG,MaXF,Gu YJ,Liu DD, Feng AN, Qian XY and Gao X: Role of epithelial-mesenchymal transition markers E-cadherin, $\mathrm{N}$-cadherin, $\beta$-catenin and ZEB2 in laryngeal squamous cell carcinoma. Oncol Lett 15: 3472-3481, 2018.

23. Cuadrado A and Nebreda AR: Mechanisms and functions of $\mathrm{p} 38$ MAPK signalling. Biochem J 429: 403-417, 2010.

24. Zhang L,Zhao S, Yuan L, Wu H, Jiang H and Luo G: Placental growth factor triggers epithelial-to-mesenchymal transition-like changes in rat type II alveolar epithelial cells: Activation of nuclear factor $\kappa \mathrm{B}$ signalling pathway. Basic Clin Pharmacol Toxicol 119: 498-504, 2016

25. Mourani PM and Abman SH: Pulmonary hypertension and vascular abnormalities in bronchopulmonary dysplasia. Clin Perinatol 42: 839-855, 2015.

26. Sun Y, Liu WZ, Liu T, Feng X, Yang N and Zhou HF: Signaling pathway of MAPK/ERK in cell proliferation, differentiation, migration, senescence and apoptosis. J Recept Signal Transduct Res 35: 600-604, 2015.

27. $\mathrm{Hu} \mathrm{X}$, Shen $\mathrm{H}$, Wang $\mathrm{Y}$ and Zhao M: Liver $\mathrm{X}$ receptor agonist TO901317 attenuates paraquat-induced acute lung injury through inhibition of NF- $\mathrm{NB}$ and JNK/p38 MAPK signal pathways. Biomed Res Int 2017: 4652695, 2017.
28. Zhang L, Zhao S, Yuan L, Wu H, Jiang H, Luo G and Zhao S: Knockdown of placental growth factor (PLGF) mitigates hyperoxia-induced acute lung injury in neonatal rats: Suppressive effects on NFkappaB signaling pathway. Int Immunopharmacol 38: 167-174, 2016.

29. Nie M, Wang Y, Lu Y, Yuan Y, Liu Y and Li X: Protective effects of fucoidan against hyperoxic lung injury via the ERK signaling pathway. Mol Med Rep 17: 1813-1818, 2018.

30. Bao XC, Fang YQ, You P, Zhang S and Ma J: Protective role of peroxisome proliferator-activated receptor beta/delta in acute lung injury induced by prolonged hyperbaric hyperoxia in rats. Respir Physiol Neurobiol 199: 9-18, 2014.

31. Porzionato A, Sfriso MM, Mazzatenta A, Macchi V, De Caro R and Di Giulio C: Effects of hyperoxic exposure on signal transduction pathways in the lung. Respir Physiol Neurobiol 209: 106-114, 2015.

(i)(9) This work is licensed under a Creative Commons Attribution-NonCommercial-NoDerivatives 4.0 International (CC BY-NC-ND 4.0) License. 